\title{
THE BEST SOBOLEV TRACE CONSTANT AS LIMIT OF THE USUAL SOBOLEV CONSTANT FOR SMALL STRIPS NEAR THE BOUNDARY
}

\author{
JOSÉ M. ARRIETA, ANÍBAL RODRÍGUEZ-BERNAL AND JULIO D. ROSSI
}

\begin{abstract}
In this paper we prove that the best constant in the Sobolev trace embedding $H^{1}(\Omega) \hookrightarrow L^{q}(\partial \Omega)$ in a bounded smooth domain can be obtained as the limit as $\varepsilon \rightarrow 0$ of the best constant of the usual Sobolev embedding $H^{1}(\Omega) \hookrightarrow L^{q}\left(\omega_{\varepsilon}, d x / \varepsilon\right)$ where $\omega_{\varepsilon}=\{x \in \Omega: \operatorname{dist}(x, \partial \Omega)<\varepsilon\}$ is a small neighborhood of the boundary. We also analyze symmetry properties of extremals of this last embedding when $\Omega$ is a ball.
\end{abstract}

\section{INTRODUCTION.}

The main goal of this article is to obtain the best Sobolev trace constant for a given domain as the limit of the usual Sobolev constant in small strips near the boundary of the domain when the width of the strip goes to zero.

Sobolev inequalities have been studied by many authors and is by now a classical subject. It at least goes back to [2], for more references see [5]. Relevant for the study of boundary value problems for differential operators is the Sobolev trace inequality that has been intensively studied, see for example, [3], [7], [8], [9], [10].

In this paper we consider the best Sobolev trace constant. Given a bounded smooth domain $\Omega \subset \mathbb{R}^{N}$, we deal with the best constant of the Sobolev trace embedding $H^{1}(\Omega) \hookrightarrow L^{q}(\partial \Omega)$. For every critical or subcritical exponent, $1 \leq q \leq 2_{*}=2(N-1) /(N-2)$, we have the Sobolev trace inequality: there exists a constant $C$ such that

$$
C\left(\int_{\partial \Omega}|v|^{q} d S\right)^{2 / q} \leq \int_{\Omega}\left(|\nabla v|^{2}+v^{2}\right) d x
$$

Key words and phrases. Nonlinear boundary conditions, Sobolev trace embedding, symmetry of extremals.

2000 Mathematics Subject Classification. 35J65, $46 \mathrm{E} 35$. 
for all $v \in H^{1}(\Omega)$. The best Sobolev trace constant is the largest $C$ such that the above inequality holds, that is,

$$
T_{q}=\inf _{v \in H^{1}(\Omega) \backslash H_{0}^{1}(\Omega)} \frac{\int_{\Omega}|\nabla v|^{2}+v^{2} d x}{\left(\int_{\partial \Omega}|v|^{q} d S\right)^{2 / q}} .
$$

For subcritical exponents, $1 \leq q<2_{*}$, the embedding is compact, so we have existence of extremals, i.e. functions where the infimum is attained. These extremals can be taken strictly positive in $\bar{\Omega}$ and smooth up to the boundary. If we normalize the extremals with

$$
\int_{\partial \Omega}|u|^{q} d S=1
$$

it follows that they are weak solutions of the following problem

$$
\begin{cases}-\Delta u+u=0 & \text { in } \Omega, \\ \frac{\partial u}{\partial \nu}=T_{q}|u|^{q-2} u & \text { on } \partial \Omega,\end{cases}
$$

where $\nu$ is the unit outward normal vector. In the special case $q=2$ (1.3) is a linear eigenvalue problem of Steklov type, see [17]. In the rest of this article we will assume that the extremals are normalized according to (1.2).

As we have mentioned, we want to see how the best trace constant, $T_{q}$, can be obtained as the limit of the usual Sobolev constant for some subdomains. To this end, let us consider the subset of $\Omega$

$$
\omega_{\varepsilon}=\{x \in \Omega: \operatorname{dist}(x, \partial \Omega)<\varepsilon\} .
$$

Notice that this set has measure $\left|\omega_{\varepsilon}\right| \sim \varepsilon|\partial \Omega|$ for small values of $\varepsilon$. For sufficiently small $\sigma \geq 0$ we can define the "parallel" interior boundary $\Gamma_{\sigma}=\{y-\sigma \nu(y), y \in \partial \Omega\}$, where $\nu(y)$ denotes the outward unitary normal at $y \in \partial \Omega$. Note that $\Gamma_{0}=\partial \Omega$. Then, we can also look at the set $\omega_{\varepsilon}$ as the neighborhood of $\Gamma_{0}$ defined by

$$
\omega_{\varepsilon}=\{x=y-\sigma \nu(y), y \in \partial \Omega, \sigma \in[0, \varepsilon)\}=\bigcup_{0 \leq \sigma<\varepsilon} \Gamma_{\sigma}
$$

for sufficiently small $\varepsilon$, say $0<\varepsilon<\varepsilon_{0}$. We also denote by $\Omega_{\delta}=\{x \in$ $\Omega: \operatorname{dist}(x, \partial \Omega)>\delta\}$ and for $\delta$ small we have that $\partial \Omega_{\delta}=\Gamma_{\delta}$.

Let us consider the usual Sobolev embedding associated to the set $\omega_{\varepsilon}$, that is,

$$
H^{1}(\Omega) \hookrightarrow L^{q}\left(\omega_{\varepsilon}, \frac{d x}{\varepsilon}\right)
$$


We have normalized the size of $\omega_{\varepsilon}$ by taking $d x / \varepsilon$ as measure in $\omega_{\varepsilon}$. In this case the embedding is continuous for exponents $q$ such that $1 \leq q \leq 2^{*}=2 N /(N-2)$. Note that $2^{*}=2 N /(N-2)$ is larger than $2_{*}=2(N-1) /(N-2)$. The best constant associated to this embedding is given by

$$
S_{q}(\varepsilon)=\inf _{v \in H^{1}(\Omega)} \frac{\int_{\Omega}|\nabla v|^{2}+v^{2} d x}{\left(\frac{1}{\varepsilon} \int_{\omega_{\varepsilon}}|v|^{q} d x\right)^{2 / q}} .
$$

For $q<2^{*}$, by compactness, the infimum is attained. The extremals, normalized by

$$
\frac{1}{\varepsilon} \int_{\omega_{\varepsilon}}|u|^{q} d x=1
$$

are weak solutions of

$$
\begin{cases}-\Delta u+u=\frac{S_{q}(\varepsilon)}{\varepsilon} \chi_{\omega_{\varepsilon}}(x)|u|^{q-2} u & \text { in } \Omega \\ \frac{\partial u}{\partial \nu}=0 & \text { on } \partial \Omega\end{cases}
$$

where $\chi_{\omega_{\varepsilon}}$ denotes the characteristic function.

Our main result is the following:

Theorem 1. Let $T_{q}$ and $S_{q}(\varepsilon)$ be the best Sobolev constants given by (1.1) and (1.4).

(1) For critical or subcritical $q, 1 \leq q \leq 2_{*}=2(N-1) /(N-2)$, we have

$$
\lim _{\varepsilon \rightarrow 0} S_{q}(\varepsilon)=T_{q}
$$

Moreover, for subcritical $q, 1 \leq q<2_{*}=2(N-1) /(N-2)$, the extremals of $S_{q}(\varepsilon)$ normalized according to (1.5) converge strongly (along subsequences) in $H^{1}(\Omega)$ and in $C^{\beta}(\Omega)$, for some $\beta>0$, to an extremal of (1.1),

$$
\lim _{\varepsilon \rightarrow 0} u_{\varepsilon}=u_{0}, \quad \text { strongly in } H^{1}(\Omega) \text { and in } C^{\beta}(\Omega) .
$$

In the critical case, $q=2_{*}=2(N-1) /(N-2)$, the extremals of $S_{q}(\varepsilon)$ converge weakly (along subsequences) in $H^{1}(\Omega)$ to a limit, $u_{0}$, that is a weak solution of (1.3). This convergence is strong in $H^{1}(\Omega)$ if and only if the limit verifies $\int_{\partial \Omega} u_{0}^{q}=1$ and in this case $u_{0}$ is an extremal for $T_{2_{*}}$. 
(2) For supercritical $q, 2_{*}=2(N-1) /(N-2)<q<2^{*}=2 N /(N-2)$, we have

$$
\lim _{\varepsilon \rightarrow 0} S_{q}(\varepsilon)=0
$$

A reference closely related to this work is [1] where the authors consider concentrated reactions near the boundary in an elliptic problem. They prove that the solutions converge to a solution of a problem with a nonhomogeneous flux condition at the boundary. Our results can be viewed as a complement of the results of [1] since here we deal with (nonlinear) eigenvalue problems when the reactions are concentrated near the boundary (see the right hand side in (1.6)).

Next, we look at the symmetry for extremals of (1.4) in the special case when $\Omega$ is a ball, $\Omega=B(0, R)$. In this case we prove the following result.

Theorem 2. Let $S_{q}(\varepsilon)$ the best Sobolev constant given by (1.4) with $\Omega=B(0, R)$.

(1) For $1 \leq q \leq 2$ and for every $R, \varepsilon>0$, the extremals of (1.4) in a ball are radial functions that do not change sign. In particular, there exists a unique non negative extremal of (1.4) satisfying (1.5).

(2) For $2<q<2_{*}=2(N-1) /(N-2)$, there exist $0<R_{0} \leq R_{1}<\infty$ such that:

(2.1) for $0<R \leq R_{0}$ and $\varepsilon$ small (possibly depending on $R$ ) the extremals of (1.4) are radial.

(2.2) for $R \geq R_{1}$ and $\varepsilon$ small (possibly depending on $R$ ) the extremals of (1.4) are not radial.

\section{Proof of Theorem 1}

This section is devoted to the proof of Theorem 1. First, we prove that the Sobolev trace constant is continuous as a function of the domain. We believe that this result has independent interest by itself.

Lemma 2.1. Let $\Omega_{\delta}=\{x \in \Omega: \operatorname{dist}(x, \partial \Omega)>\delta\}$. Then the function

$$
\delta \rightarrow T_{q}\left(\Omega_{\delta}\right)
$$

is continuous at $\delta=0$.

Proof. Consider a fixed $\varepsilon_{0}>0$ small enough. For all $0<\delta<\varepsilon_{0}$, let us consider a smooth increasing function $\psi_{\delta}$ such that $\psi_{\delta}(0)=\delta$, 
$\psi_{\delta}(s)=s$ for all $s \geq \varepsilon_{0}$ and $\psi_{\delta}(s) \rightarrow s$ as $\delta \rightarrow 0$ in $C^{1}([0, \infty))$. Now we take the diffeomorphism

$$
\begin{gathered}
A_{\delta}: \Omega \rightarrow \Omega_{\delta}, \\
A_{\delta}(x)= \begin{cases}y-\psi_{\delta}(s) \nu(y) & \text { for } x=y-s \nu(y) \in \omega_{\varepsilon}, s \in(0, \varepsilon), \\
x & \text { for } x \in \Omega \backslash \overline{\omega_{\varepsilon}} .\end{cases}
\end{gathered}
$$

which is also a diffeomorphism when restricted to the boundary,

$$
A_{\delta}: \partial \Omega \rightarrow \partial \Omega_{\delta}
$$

This diffeomorphism has bounded derivatives and moreover

$$
\lim _{\delta \rightarrow 0}\left\|D A_{\delta}(x)-I\right\|=0,
$$

uniformly in $\bar{\Omega}$. Here $I \in \mathcal{M}_{n \times n}$ is the identity matrix.

Therefore, we can change variables with,

$$
u(x)=v\left(A_{\delta}(x)\right),
$$

for $x \in \Omega$ or $x \in \partial \Omega$. This induces a map, that we denote the same

$$
A_{\delta}: H^{1}(\Omega) \mapsto H^{1}\left(\Omega_{\delta}\right)
$$

which is a diffeomorphism. Moreover, we have that the following diagram is commutative

$$
\begin{array}{ccc}
H^{1}(\Omega) & \rightarrow & L^{q}(\partial \Omega) \\
A_{\delta} \downarrow & & \downarrow A_{\delta} \\
H^{1}\left(\Omega_{\delta}\right) & \rightarrow & L^{q}\left(\partial \Omega_{\delta}\right) .
\end{array}
$$

Therefore, from (2.1), we obtain

$$
C_{1}(\delta) \int_{\Omega}|\nabla u|^{2}+u^{2} d x \leq \int_{\Omega_{\delta}}|\nabla v|^{2}+v^{2} d x \leq C_{2}(\delta) \int_{\Omega}|\nabla u|^{2}+u^{2} d x,
$$

where $C_{i}(\delta) \rightarrow 1$ as $\delta \rightarrow 0$.

In a similar way, we get

$$
C_{1}(\delta) \int_{\partial \Omega}|u|^{q} d S \leq \int_{\partial \Omega_{\delta}}|v|^{q} d S \leq C_{2}(\delta) \int_{\partial \Omega}|u|^{q} d S
$$

with $C_{i}(\delta) \rightarrow 1$ as $\delta \rightarrow 0$.

From the previous inequalities we obtain that there exist two constants $K_{1}, K_{2}$ such that $K_{i}(\delta) \rightarrow 1$ as $\delta \rightarrow 0$ and

$$
K_{1}(\delta) T_{q}(\Omega) \leq T_{q}\left(\Omega_{\delta}\right) \leq K_{2}(\delta) T_{q}(\Omega) .
$$

The desired continuity is proved. 
The next result shows that the traces on $\partial \Omega_{\delta}$ also behave continuously as $\delta \rightarrow 0$. In order to do this, we first figure out a device that allows to compare traces taken on different surfaces close to the boundary of $\Omega$. For this observe that, for any $q \leq 2_{*}$ we can define the mapping

$$
\gamma_{\delta}: H^{1}(\Omega) \rightarrow L^{q}\left(\partial \Omega_{\delta}\right) \leftrightarrow L^{q}(\partial \Omega)
$$

Here the first arrow denotes traces and the second one denotes the diffeomorphism induced by $A_{\delta}^{-1}$ as in (2.2).

Then, we have the following result, which in particular complements some results in [1].

Lemma 2.2. Denoting by $\gamma$ the trace operator on $\partial \Omega$, we have

$$
\lim _{\delta \rightarrow 0} \gamma_{\delta}=\gamma \quad \text { in } \quad L^{q}(\partial \Omega)
$$

on compact sets of $H^{1}(\Omega)$ if $q=2_{*}$ or in $\mathcal{L}\left(H^{1}(\Omega), L^{q}(\partial \Omega)\right)$ if $q<2_{*}$.

In particular, for $q \leq 2_{*}$, if $u_{\varepsilon}$ is a bounded sequence in $H^{1}(\Omega)$, then $\frac{1}{\varepsilon} \int_{\omega_{\varepsilon}}\left|u_{\varepsilon}\right|^{q}$ is also bounded.

Moreover, if $u_{\varepsilon} \rightarrow u_{0}$ strongly in $H^{1}(\Omega)$ and $q \leq 2_{*}$, then

$$
\int_{\partial \Omega_{\delta(\varepsilon)}}\left|u_{\varepsilon}\right|^{q} d S \rightarrow \int_{\partial \Omega}\left|u_{0}\right|^{q} d S
$$

as $\delta(\varepsilon) \rightarrow 0$ and

$$
\frac{1}{\varepsilon} \int_{\omega_{\varepsilon}}\left|u_{\varepsilon}\right|^{q} d x \rightarrow \int_{\partial \Omega}\left|u_{0}\right|^{q} d S
$$

as $\varepsilon \rightarrow 0$.

Proof. If $q \leq 2_{*}$ and $u_{\varepsilon}$ is a bounded sequence in $H^{1}(\Omega)$, we write

$$
\begin{aligned}
& \frac{1}{\varepsilon} \int_{\omega_{\varepsilon}}\left|u_{\varepsilon}\right|^{q} d x=\frac{1}{\varepsilon} \int_{0}^{\varepsilon} \int_{\partial \Omega_{\delta}}\left|u_{\varepsilon}\right|^{q} d S d \delta \leq \\
& \frac{1}{\varepsilon} \int_{0}^{\varepsilon} T_{q}\left(\Omega_{\delta}\right)^{-\frac{q}{2}}\left\|u_{\varepsilon}\right\|_{H^{1}\left(\Omega_{\delta}\right)}^{q} d \delta \leq \sup _{\delta \in[0, \varepsilon]}\left[T_{q}\left(\Omega_{\delta}\right)^{-\frac{q}{2}}\right]\left\|u_{\varepsilon}\right\|_{H^{1}(\Omega)}^{q}
\end{aligned}
$$

which is bounded using Lemma 2.1 and the fact that the sequence $u_{\varepsilon}$ is bounded in $H^{1}(\Omega)$.

Note that if $q<2_{*}$ there exists some $0<s<1$, such that

$$
\gamma_{\delta}: H^{1}(\Omega) \hookrightarrow H^{s}(\Omega) \rightarrow L^{q}\left(\partial \Omega_{\delta}\right) \leftrightarrow L^{q}(\partial \Omega) .
$$

In a similar fashion, if $q=2_{*}$, we take $s=1$. 
For any fixed $u \in H^{s}(\Omega)$, from (2.3), we have that these operators converge to the usual trace on $\partial \Omega$, that is

$$
\lim _{\delta \rightarrow 0} \gamma_{\delta}(u)=\gamma(u)
$$

Moreover, we have

$$
\left\|\gamma_{\delta}\right\|_{\mathcal{L}\left(H^{s}(\Omega), L^{q}(\partial \Omega)\right)} \leq C
$$

uniformly on $\delta$. Hence, from the Banach-Alouglu-Bourbaki lemma, we get

$$
\lim _{\delta \rightarrow 0} \gamma_{\delta}=\gamma
$$

on compact sets of $H^{s}(\Omega)$.

In addition, if $u_{\varepsilon} \rightarrow u_{0}$ strongly in $H^{1}(\Omega)$

$$
\lim _{\varepsilon \rightarrow 0} \int_{\partial \Omega}\left|\gamma_{\varepsilon}\left(u_{\varepsilon}\right)\right|^{q} d S=\int_{\partial \Omega}\left|u_{0}\right|^{q} d S
$$

which combined with (2.3) gives (2.4).

On the other hand, to obtain (2.5) we write

$$
\frac{1}{\varepsilon} \int_{\omega_{\varepsilon}}\left|u_{\varepsilon}\right|^{q} d x=\frac{1}{\varepsilon} \int_{0}^{\varepsilon} \int_{\partial \Omega_{\delta}}\left|u_{\varepsilon}\right|^{q} d S d \delta .
$$

Since for every $\delta<\varepsilon, \int_{\partial \Omega_{\delta}}\left|u_{\varepsilon}\right|^{q}$ and $\int_{\partial \Omega}\left|u_{0}\right|^{q}$ are uniformly close, we get $(2.5)$.

Remark 2.3. The only property that we have actually used in the proof of the previous results is (2.1). Therefore both lemmas above remain true for any family of domains $\Omega_{\delta}$ such that there exists a diffeomorphism $A_{\delta}: \Omega \mapsto \Omega_{\delta}$ with $A_{\delta}: \partial \Omega \mapsto \partial \Omega_{\delta}$ such that (2.1) holds. Also note that in Lemma 2.2 the conclusions remain true for $q<2_{*}$ under the weaker assumption of convergence in $H^{s}(\Omega)$ for $s<1$ but close enough to 1.

Proof of Theorem 1. We first prove (1.7) for critical or subcritical exponents, i.e. $1 \leq q \leq 2_{*}=2(N-1) /(N-2)$. Given $k>0$, let us take a regular function $u_{k}$ such that

$$
T_{q}+\frac{1}{k} \geq \frac{\int_{\Omega}\left|\nabla u_{k}\right|^{2}+u_{k}^{2} d x}{\left(\frac{1}{\varepsilon} \int_{\omega_{\varepsilon}} u_{k}^{q} d x\right)^{2 / q}} .
$$


By the regularity of $u_{k}$, from Lemma 2.2 (see also [1]), we have, for a fixed $k$,

$$
\lim _{\varepsilon \rightarrow 0} \frac{1}{\varepsilon} \int_{\omega_{\varepsilon}} u_{k}^{q} d x=\lim _{\varepsilon \rightarrow 0} \frac{1}{\varepsilon} \int_{0}^{\varepsilon} \int_{\Gamma_{s}} u_{k}^{q} d \bar{x} d s=\int_{\partial \Omega} u_{k}^{q} d \bar{x} .
$$

Therefore, using $u_{k}$ as test in (1.4) and taking limits we get

$$
\limsup _{\varepsilon \rightarrow 0} S_{q}(\varepsilon) \leq T_{q}+\frac{1}{k}
$$

Letting $k \rightarrow \infty$ we obtain

$$
\limsup _{\varepsilon \rightarrow 0} S_{q}(\varepsilon) \leq T_{q}
$$

Now let us prove that for $q \leq 2_{*}$ we have

$$
\liminf _{\varepsilon \rightarrow 0} S_{q}(\varepsilon) \geq T_{q}
$$

For this, note that for $u \in H^{1}(\Omega)$ we get, using the restriction to $\Omega_{\delta}$,

$$
\left(\int_{\partial \Omega_{\delta}}|u|^{q} d S\right)^{2 / q} \leq \frac{1}{T_{q}\left(\Omega_{\delta}\right)}\|u\|_{H^{1}\left(\Omega_{\delta}\right)}^{2} \leq \frac{1}{T_{q}\left(\Omega_{\delta}\right)}\|u\|_{H^{1}(\Omega)}^{2} .
$$

Integrating for $\delta \in(0, \varepsilon)$ we obtain

$$
\frac{1}{\varepsilon} \int_{\omega_{\varepsilon}}|u|^{q} d x=\frac{1}{\varepsilon} \int_{0}^{\varepsilon} \int_{\partial \Omega_{\delta}}|u|^{q} d \delta \leq\left(\frac{1}{\varepsilon} \int_{0}^{\varepsilon} \frac{d \delta}{\left(T_{q}\left(\Omega_{\delta}\right)\right)^{q / 2}}\right)\|u\|_{H^{1}(\Omega)}^{q} .
$$

Thus, we have obtained

$$
\left(\frac{1}{\varepsilon} \int_{0}^{\varepsilon} \frac{d \delta}{\left(T_{q}\left(\Omega_{\delta}\right)\right)^{q / 2}}\right)^{-2 / q} \leq S_{q}(\varepsilon) .
$$

This fact, together with the continuity of the map

$$
\delta \rightarrow T_{q}\left(\Omega_{\delta}\right)
$$

proved in Lemma 2.1, gives (2.7).

From (2.6) and (2.7) we obtain

$$
\lim _{\varepsilon \rightarrow 0} S_{q}(\varepsilon)=T_{q},
$$

as we wanted to prove.

Now we turn our attention to the convergence of extremals in the subcritical case $q<2_{*}$. To prove this fact, let us consider $u_{\varepsilon}$ an extremal of $S_{q}(\varepsilon)$ normalized by

$$
\frac{1}{\varepsilon} \int_{\omega_{\varepsilon}}\left|u_{\varepsilon}\right|^{q} d x=1
$$


Hence we have, for $\varepsilon$ small, using (2.6),

$$
\left\|u_{\varepsilon}\right\|_{H^{1}(\Omega)}^{2}=S_{q}(\varepsilon) \leq T_{q}+1 \text {. }
$$

Therefore the sequence $u_{\varepsilon}$ is bounded in $H^{1}(\Omega)$ and we can extract a subsequence (that we still denote $u_{\varepsilon}$ ) such that

$$
\begin{array}{ll}
u_{\varepsilon} \rightarrow u_{0} & \text { weakly in } H^{1}(\Omega), \\
u_{\varepsilon} \rightarrow u_{0} & \text { strongly in } L^{2}(\Omega), \\
u_{\varepsilon} \rightarrow u_{0} & \text { strongly in } H^{s}(\Omega), \forall s<1, \\
u_{\varepsilon} \rightarrow u_{0} & \text { strongly in } L^{q}(\partial \Omega), \\
u_{\varepsilon} \rightarrow u_{0} & \text { a.e. in } \Omega .
\end{array}
$$

Now we claim that,

$$
\int_{\partial \Omega}\left|u_{0}\right|^{q} d S=1
$$

To prove this, note that as we have

$$
1=\frac{1}{\varepsilon} \int_{\omega_{\varepsilon}}\left|u_{\varepsilon}\right|^{q} d x=\frac{1}{\varepsilon} \int_{0}^{\varepsilon} \int_{\partial \Omega_{\delta}}\left|u_{\varepsilon}\right|^{q} d S d \delta
$$

from the integral mean value theorem, there exists $0 \leq \delta(\varepsilon) \leq \varepsilon$ such that

$$
\int_{\partial \Omega_{\delta}}\left|u_{\varepsilon}\right|^{q} d S=1
$$

Now, from the convergence of $u_{\varepsilon}$ to $u_{0}$ in $H^{s}(\Omega)$, valid for $0<s<1$, we conclude that

$$
\int_{\partial \Omega}\left|u_{0}\right|^{q} d S=1
$$

see the Remark after Lemma 2.2. This finishes the proof of the claim.

With this in mind, we have

$$
\begin{aligned}
T_{q} & \leq \frac{\int_{\Omega}\left|\nabla u_{0}\right|^{2}+u_{0}^{2} d x}{\left(\int_{\partial \Omega}\left|u_{0}\right|^{q} d S\right)^{2 / q}} \leq\left\|u_{0}\right\|_{H^{1}(\Omega)}^{2} \leq \liminf _{\varepsilon \rightarrow 0}\left\|u_{\varepsilon}\right\|_{H^{1}(\Omega)}^{2} \\
& \leq \limsup _{\varepsilon \rightarrow 0}\left\|u_{\varepsilon}\right\|_{H^{1}(\Omega)}^{2}=\limsup _{\varepsilon \rightarrow 0} S_{q}(\varepsilon)=T_{q} .
\end{aligned}
$$

Therefore

$$
\lim _{\varepsilon \rightarrow 0}\left\|u_{\varepsilon}\right\|_{H^{1}(\Omega)}=\left\|u_{0}\right\|_{H^{1}(\Omega)} .
$$

In particular, the convergence of the norms implies that the extremals of $S_{q}(\varepsilon)$ normalized according to $(2.9)$ converge strongly in $H^{1}(\Omega)$ to 
an extremal of (1.1),

$$
\lim _{\varepsilon \rightarrow 0} u_{\varepsilon}=u_{0}, \quad \text { strongly in } H^{1}(\Omega)
$$

which satisfies (2.11).

Now, let us prove that we have convergence in $C^{\beta}(\Omega)$, for some $\beta>0$. To this end we will use some results from [1] that describe the behavior of solutions of linear elliptic equations with concentrated potentials.

Denote by $V_{\varepsilon}(x)=S_{q}(\varepsilon) u_{\varepsilon}^{q-2}$ so that $u_{\varepsilon}$ is a solution of the problem

$$
\begin{cases}-\Delta u_{\varepsilon}+u_{\varepsilon}=\frac{1}{\varepsilon} \chi_{\omega_{\varepsilon}} V_{\varepsilon} u_{\varepsilon} & \text { in } \Omega \\ \frac{\partial u_{\varepsilon}}{\partial \nu}=0 & \text { on } \partial \Omega .\end{cases}
$$

First, remark that as $q$ is subcritical we can choose $r>N-1$ such that

$$
\frac{1}{\varepsilon} \int_{\omega_{\varepsilon}}\left|V_{\varepsilon}\right|^{r} d x=\frac{S_{q}(\varepsilon)^{r}}{\varepsilon} \int_{\omega_{\varepsilon}}\left|u_{\varepsilon}\right|^{(q-2) r} d x \leq C,
$$

with $C$ independent of $\varepsilon$. Indeed, as $u_{\varepsilon}$ is uniformly bounded in $H^{1}(\Omega)$ we have from Lemma 2.2 , that for any $\theta \leq 2(N-1) /(N-2)$,

$$
\frac{1}{\varepsilon} \int_{\omega_{\varepsilon}}\left|u_{\varepsilon}\right|^{\theta} d x \leq C
$$

Now, just write $\theta=(q-2) r$ and use the fact that $q<2(N-1) /(N-2)$ (this implies $(q-2)<2 /(N-2))$ to obtain that for some $r>N-1$ we have $(q-2) r \leq 2(N-1) /(N-2)$.

Moreover, since $S_{q}(\varepsilon) \rightarrow T_{q}, u_{\varepsilon} \rightarrow u_{0}$ in $H^{1}(\Omega)$ and $q$ is subcritical, we have that

$$
\frac{1}{\varepsilon} \int_{\omega_{\varepsilon}} V_{\varepsilon} \phi d x \rightarrow \int_{\partial \Omega} V_{0} \phi d S
$$

for any smooth function $\phi$, where $V_{0}(x)=T_{q} u_{0}^{q-2}(x)$. Hence, $u_{0}$ satisfies

$$
\begin{cases}-\Delta u_{0}+u_{0}=0 & \text { in } \Omega, \\ \frac{\partial u_{0}}{\partial \nu}=V_{0} u_{0} & \text { on } \partial \Omega .\end{cases}
$$

With all this at hand, we can apply Theorem 3.1 and Corollary 3.2 from [1], that guarantee the convergence in the Hölder norm $C^{\beta}(\Omega)$, for some $\beta>0$.

In the critical case $q=2_{*}$ we also obtain a uniform bound in $H^{1}(\Omega)$ for the extremals $u_{\varepsilon}$ of $S_{q}(\varepsilon)$. Therefore we can extract a subsequence such that (2.10) holds. Passing to the limit in the weak form of (1.6) we get that the limit $u_{0}$ is a weak solution of (1.3). However, due to 
the lack of compactness, we cannot ensure that $u_{0}$ verifies $\int_{\partial \Omega}\left|u_{0}\right|^{q}=1$ in this case.

To finish the proof of the theorem it remains to show (1.8) in the supercritical case $2_{*}=2(N-1) /(N-2)<q<2^{*}=2 N /(N-2)$. To see this fact assume that $0 \in \partial \Omega$ and consider

$$
u(x)=|x|^{-\lambda} .
$$

Where we choose $\lambda$ such that $u \in H^{1}(\Omega)$, i.e. $\lambda<(N-2) / 2$. Now we choose $\lambda=\lambda(q)$ such that

$$
\int_{\partial \Omega}|u|^{q} d S=+\infty
$$

that is, $\lambda \geq(N-1) / q$, which is possible since $q>2_{*}$. We observe that with this choice, we have

$$
\lim _{\varepsilon \rightarrow 0} \frac{1}{\varepsilon} \int_{\omega_{\varepsilon}}|u|^{q} d x=+\infty .
$$

The proof is finished.

Remark 2.4. Observe that in the critical case, using a sequence of minimizers and subsequences if necessary we have $u_{\varepsilon} \rightarrow u_{0}$ weakly in $H^{1}(\Omega)$ and $S_{\varepsilon}(q) \rightarrow T_{q}$. Also, we have

$$
\left\|u_{0}\right\|_{H^{1}(\Omega)}^{2} \leq \liminf _{\varepsilon \rightarrow 0}\left\|u_{\varepsilon}\right\|_{H^{1}(\Omega)}^{2} \leq \limsup _{\varepsilon \rightarrow 0}\left\|u_{\varepsilon}\right\|_{H^{1}(\Omega)}^{2}=\limsup _{\varepsilon \rightarrow 0} S_{q}(\varepsilon)=T_{q}
$$

and

$$
T_{q} \leq \frac{\int_{\Omega}\left|\nabla u_{0}\right|^{2}+u_{0}^{2} d x}{\left(\int_{\partial \Omega}\left|u_{0}\right|^{q} d S\right)^{2 / q}}
$$

Hence if $u_{0}$ is a minimizer, then $\int_{\partial \Omega}\left|u_{0}\right|^{q} d S \leq 1$. Conversely, if $\int_{\partial \Omega}\left|u_{0}\right|^{q} d S \geq 1$ then the argument above shows that this integral is actually equal to 1 and $u_{0}$ is a minimizer. Moreover in such a case, we get the convergence of the $H^{1}(\Omega)$ norms and hence the strong convergence in this space.

Thus, $u_{0}$ is a minimizer if and only if $\int_{\partial \Omega}\left|u_{0}\right|{ }^{q} d S=1$ which in turn is equivalent to the strong convergence.

Also, in the critical case it may happen then that one has (1.5) and $\int_{\partial \Omega}\left|u_{0}\right|^{q} d S<1$. 


\section{Proof of Theorem 2}

We divide the proof of Theorem 2 in several lemmas. Along this section we take $\Omega=B(0, R)$, except in the next result.

Lemma 3.1. Let $\Omega$ be arbitrary. Then for any $1 \leq q \leq 2$ and any $\varepsilon>0$ every extremal is of constant sign. Moreover, there exists a unique positive extremal of (1.4), normalized according to (1.5).

Proof. Note that non negative extremals of (1.4) are indeed positive solutions of (1.6), i.e. they satisfy, when normalized as in (1.5),

$$
\begin{cases}-\Delta u=f(x, u)=a(x) u^{\rho}-u & \text { in } \Omega, \\ \frac{\partial u}{\partial \nu}=0 & \text { on } \partial \Omega,\end{cases}
$$

where $a(x)=\frac{S_{q}(\varepsilon)}{\varepsilon} \chi_{\omega_{\varepsilon}}(x) \geq 0$ and $\rho=q-1$. Also, note that from (1.4), non negative extremals exists, since the absolute value of an extremal is an extremal.

Now, we use an argument from [18]; see also [15] and [16]. Note that if $q<2$ then $\rho<1$. Hence, if $x \in \Omega \backslash \overline{\omega_{\varepsilon}}$ we have $f(x, u)=-u \leq$ $C(x) u+D(x)$ if we take $C(x)=-1$ and $D(x)=0$.

On the other hand, if $x \in \omega_{\varepsilon}$ Young's inequality yields for sufficiently small $\delta$,

$$
f(x, u) \leq(\delta-1) u+\beta\left[\frac{S_{q}(\varepsilon)}{\delta^{\rho} \varepsilon}\right]^{\frac{1}{1-\rho}}
$$

for some constant $\beta>0$ and we can take $C(x)=\delta-1$ and

$$
D(x)=\beta\left[\frac{S_{q}(\varepsilon)}{\delta^{\rho} \varepsilon}\right]^{\frac{1}{1-\rho}} .
$$

In summary

$$
C(x)=\delta \chi_{\omega_{\varepsilon}}(x)-1, \quad D(x)=\beta\left[\frac{S_{q}(\varepsilon)}{\delta^{\rho} \varepsilon}\right]^{\frac{1}{1-\rho}} \chi_{\omega_{\varepsilon}}(x)
$$

and we have, for $u>0$ and $x \in \Omega$,

$$
f(x, u) \leq C(x) u+D(x) .
$$

Note that for sufficiently small $\delta$, the semigroup generated by $\Delta+$ $C(x)$ in $\Omega$ with Neumann boundary conditions decays exponentially. Then since $D \in L^{\infty}(\Omega)$, we get from [18] and [15], that there exist a solution of (3.1), which is maximal in the sense of pointwise ordering. In particular it is nonzero since it bounds above in a pointwise sense any normalized positive extremal. 
Now, the proof concludes by showing that in fact (3.1) has a unique solution, which follows from the fact that

$$
\frac{f(x, u)}{u}=\frac{a(x)}{u^{1-\rho}}-1
$$

is nonicreasing for $u>0$ and strictly decreasing on a set of positive measure. Indeed, let $\varphi$ be the maximal positive solution of (3.1) and $0<\psi \leq \varphi$ any other solution. Then, multiplying the equation satisfied by $\varphi$ by $\psi$ and the one for $\psi$ by $\varphi$, substracting and integrating by parts in $\Omega$, we have

$$
0=\int_{\Omega} \frac{f(x, \varphi)}{\varphi} \varphi \psi-\int_{\Omega} \frac{f(x, \psi)}{\psi} \varphi \psi=\int_{\Omega}\left(\frac{f(x, \varphi)}{\varphi}-\frac{f(x, \psi)}{\psi}\right) \varphi \psi .
$$

Now, since $\psi \leq \varphi$ we have that $\frac{f(x, \varphi)}{\varphi}-\frac{f(x, \psi)}{\psi} \leq 0$ and is non zero in a set of positive measure. Therefore, we must have $\psi \equiv 0$.

When $q=2$ the conclusion of the lemma follows easily since the first eigenvalue of the elliptic problem (1.6) is simple, [14]. Therefore there exists a unique positive eigenfunction such that (1.5) holds.

With this, if $\Omega=B(0, R)$, we get the following result, which actually proves the first part of Theorem 2 .

Corollary 3.2. For every $1 \leq q \leq 2$ and every $R, \varepsilon>0$ every extremal of (1.4) is radial and does not change sign in $\Omega$.

Proof. Note that in any case $q<2$ or $q=2$, the absolute value of an extremal is also an extremal. Therefore, the absolute value is a nonnegative extremal and must be then coincide with the unique positive extremal. This one, in turn, must be radial, since, by uniqueness, it must coincide with any rotation of it.

The following lemma proves (2.1) in Theorem 2 .

Lemma 3.3. For $2<q<2_{*}=2(N-1) /(N-2)$ there exists $R_{1}$ such that for every $R>R_{1}$ there exists $\varepsilon_{0}$ such that the extremals (1.4) are not radial for $\varepsilon<\varepsilon_{0}$.

Proof. The results of [6] imply that in this case the extremals of the best Sobolev trace constant $T_{q}(B(0, R))$ are not radial (since they develop a concentration phenomena). Since the extremals for $S_{q}(\varepsilon)$ converge to the extremals of $T_{q}(B(0, R))$ as $\varepsilon \rightarrow 0$ they cannot be radial for $\varepsilon$ small enough (possibly depending on $R$ ).

Now we finish the proof of Theorem 2 . 
Lemma 3.4. For $2<q<2_{*}=2(N-1) /(N-2)$ there exists $R_{0}$ such that for every $R \leq R_{0}$ there exists $\varepsilon_{0}$ such that there exists a radial extremal of (1.4) for $\varepsilon<\varepsilon_{0}$.

Proof. First, let us choose $R_{0}$ in such a way that for any $R<R_{0}$ the problem

$$
\begin{cases}-\Delta u+R^{2} u=0 & \text { in } B(0,1), \\ \frac{\partial u}{\partial \nu}=R^{2} \frac{T_{q}(R)}{R^{\beta}} u^{q-1} & \text { on } \partial B(0,1),\end{cases}
$$

has a unique positive solution close to $u_{0} \equiv 1$ normalized with the usual constraint $\int_{\partial B(0,1)} u^{q}=1$, see [8]. Here

$$
\beta=\frac{q N-2 N+2}{q} .
$$

Observe that the above problem is just (1.3) (together with (1.2)) rescaled from the ball of radius $R$ to the ball of radius one. Also note that, from the results of [9], we have

$$
\lim _{R \rightarrow 0} \frac{T_{q}(R)}{R^{\beta}}=\frac{|B(0,1)|}{|\partial B(0,1)|^{2 / q}} .
$$

Moreover, we can assume (taking $R_{0}$ smaller if necessary) that for $R<R_{0}$ the linearized of (3.2) is invertible. This can be obtained since for small $R$ there is a unique solution to (3.2) with $\int_{\partial B(0,1)} u^{q}=1$ and the linearized problem is invertible at $R=0, u=\frac{1}{|\partial B(0,1)|^{1 / q}}$ and then invertible at $\left(R, u_{R}\right)$ for small $R$ (see [8] for the details).

Now we want to use the implicit function theorem in (1.4). To this end, let us rescale (1.6) to the unit ball defining $v(x)=R^{\alpha} u(R x)$ where $u$ is the solution of (1.6) satisfying (1.5). If $\alpha=(N-1) / q$, we have that $v$ satisfies

$$
\frac{1}{\varepsilon R^{-1}} \int_{\Delta_{\varepsilon, R}}|v|^{q} d x=1,
$$

where $\Delta_{\varepsilon, R}=B(0,1) \backslash B\left(0,1-\varepsilon R^{-1}\right)$ and also

$$
\begin{cases}-\Delta v+R^{2} v=R^{2} \frac{S_{q}(\varepsilon)}{R^{\beta} \varepsilon R^{-1}} \chi_{\varepsilon, R}(x) v^{q-1} & \text { in } B(0,1), \\ \frac{\partial v}{\partial \nu}=0 & \text { on } \partial B(0,1),\end{cases}
$$


where $\chi_{\varepsilon, R}(x)$ is the characteristic function of $\Delta_{\varepsilon, R}$. Let

$$
S=\left\{v \in H^{1}(B(0,1)) ; \int_{\partial B(0,1)}|v|^{q} d S=1\right\} .
$$

If we multiply $v$ by an adequate constant $\mu$ in order to have $w=$ $\mu v \in S$, we have $\mu=\left(\int_{\partial B(0,1)} v^{q}\right)^{-\frac{1}{q}}$ and we are left with a solution of

$$
\begin{cases}-\Delta w+R^{2} w=R^{2} \frac{\tilde{A}(\varepsilon)}{\varepsilon R^{-1} R^{\beta}} \chi_{\varepsilon, R}(x) w^{q-1} & \text { in } B(0,1), \\ \frac{\partial w}{\partial \nu}=0 & \text { on } \partial B(0,1) .\end{cases}
$$

Here

$$
\tilde{A}(\varepsilon)=S_{q}(\varepsilon)\left(\int_{\partial B(0,1)} v^{q} d S\right)^{1-2 / q}
$$

where the integral term also depends on $\varepsilon$ through $v$. From (1.7) and the convergence of the extremals in Theorem 1, we get, using (3.3) and Lemma 2.2, that

$$
\tilde{A}(\varepsilon) \rightarrow T_{q}
$$

as $\varepsilon \rightarrow 0$.

Let us consider the functional

$$
F: S \times\left[0, \varepsilon_{0}\right] \mapsto\left(H^{1}(B(0,1))\right)^{*},
$$

given by

$$
\begin{aligned}
F(w, \varepsilon)(\phi)= & \int_{B(0,1)} \nabla w \nabla \phi d x+R^{2} \int_{B(0,1)} w \phi d x \\
& -\frac{R^{2} \tilde{A}(\varepsilon)}{\varepsilon R^{-1} R^{\beta}} \int_{B(0,1) \backslash B\left(0,1-\varepsilon R^{-1}\right)} w^{q-1} \phi d x .
\end{aligned}
$$

This functional is $C^{1}$ with respect to $w \in S$ (since $q>2$ ).

Remark that we are looking for pairs $(w, \varepsilon)$ that are solutions of $F(w, \varepsilon)=0$ (these are weak solutions of (3.4)).

To apply the implicit function theorem we need to compute

$$
\frac{\partial F}{\partial w}(u, 0)
$$

First, let us compute the derivative

$$
\begin{aligned}
\frac{\partial F}{\partial w}(w, \varepsilon)(\phi)(\chi)= & \int_{B(0,1)} \nabla \chi \nabla \phi d x+R^{2} \int_{B(0,1)} \chi \phi d x \\
& -\frac{R^{2} \tilde{A}(\varepsilon)}{\varepsilon R^{-1} R^{\beta}} \int_{B(0,1) \backslash B\left(0,1-\varepsilon R^{-1}\right)}(q-1) w^{q-2} \phi \chi d x .
\end{aligned}
$$


Taking the limit as $\varepsilon \rightarrow 0$ and evaluating at $w=u$ we obtain (by [1] or by the results of the previous section)

$$
\begin{aligned}
\frac{\partial F}{\partial w}(u, 0)(\phi)(\chi)= & \int_{B(0,1)} \nabla \chi \nabla \phi d x+R^{2} \int_{B(0,1)} \chi \phi d x \\
& -R^{2} \frac{T_{q}}{R^{\beta}} \int_{\partial B(0,1)}(q-1) u^{q-2} \phi \chi d x
\end{aligned}
$$

This problem corresponds exactly with the linearized of (3.2) that is invertible by our choice $R<R_{0}$.

Therefore, by the implicit function theorem, we get that there exists $\varepsilon_{0}$ such that for any $\varepsilon<\varepsilon_{0}$ there exists a unique solution $w_{\varepsilon} \in S$ of

$$
F\left(w_{\varepsilon}, \varepsilon\right)=0
$$

close to $u$, that is, a unique weak solution of (3.4), with

$$
\lim _{\varepsilon \rightarrow 0} w_{\varepsilon}=u \text {. }
$$

Since we have proved that every extremal of (1.4) goes to $u$ as $\varepsilon \rightarrow 0$ and we have uniqueness of solutions of (3.4) in a neighborhood of $u$, then the extremals must be radial.

Acknowledgements. The first and second authors have been partially supported by Grant BFM2003-03810, Spain and the third author by EX066, CONICET and ANPCyT PICT No. 05009, Argentina.

\section{REFERENCES}

[1] J. M. Arrieta, A. Jimenez-Casas and A. Rodriguez-Bernal. Nonhomogeneous flux condition as limit of concentrated reactions. Preprint

[2] T. Aubin. Équations différentielles non linéaires et le problème de Yamabe concernant la courbure scalaire. J. Math. Pures et Appl., 55 (1976), 269-296.

[3] R.J. Biezuner. Best constants in Sobolev trace inequalities. Nonlinear Analysis, 54 (2003), 575-589.

[4] P. Cherrier, Problèmes de Neumann non linéaires sur les variétés Riemanniennes. J. Funct. Anal. Vol. 57 (1984), 154-206.

[5] O. Druet and E. Hebey. The AB program in geometric analysis: sharp Sobolev inequalities and related problems. Mem. Amer. Math. Soc. 160 (761) (2002).

[6] M. del Pino and C. Flores. Asymptotic behavior of best constants and extremals for trace embeddings in expanding domains. Comm. Partial Differential Equations, 26 (11-12) (2001), 2189-2210.

[7] J. F. Escobar. Sharp constant in a Sobolev trace inequality. Indiana Univ. Math. J., 37 (3) (1988), 687-698. 
[8] J. Fernández Bonder, E. Lami Dozo and J.D. Rossi. Symmetry properties for the extremals of the Sobolev trace embedding. Ann. Inst. H. Poincaré. Anal. Non Linéaire, 21 (2004), no. 6, 795-805.

[9] J. Fernández Bonder and J.D. Rossi. Asymptotic behavior of the best Sobolev trace constant in expanding and contracting domains. Comm. Pure Appl. Anal., 1 (3) (2002), 359-378.

[10] J. Fernández Bonder and J.D. Rossi. on the existence of extremals for the Sobolev trace embedding theorem with critical exponent. Bull. London Math. Soc., 37(1) (2005), 119-125.

[11] O. Ladyzhenskaya and N. Uralseva. "Linear and Quasilinear Elliptic Equations", Academic Press (1968).

[12] E. Lami Dozo and O. Torne, Symmetry and symmetry breaking for minimizers in the trace inequality. Comm. Contemp. Math., 7(6) (2005), 727-746.

[13] Y. Li and M. Zhu. Sharp Sobolev trace inequalities on Riemannian manifolds with boundaries. Comm. Pure Appl. Math., 50 (1997), 449-487.

[14] S. Martinez and J.D. Rossi. Isolation and simplicity for the first eigenvalue of the p-laplacian with a nonlinear boundary condition. Abst. Appl. Anal., 7 (5), (2002), 287-293.

[15] A. Rodríguez-Bernal and A. Vidal-Lopez. Extremal equilibria for parabolic nonlinear reaction diffusion equations. Proceedings of the Equadiff 2005, Bratislava, Eslovakia.

[16] A. Rodríguez-Bernal and A. Vidal-Lopez. Extremal equilibria for nonlinear parabolic equations and applications. Universidad Complutense de Madrid. Preprint MA-UCM-2006-6.

[17] M. W. Steklov, Sur les problèmes fondamentaux en physique mathématique, Ann. Sci. Ecole Norm. Sup., Vol. 19 (1902), 455-490.

[18] A. Vidal-Lopez, Soluciones extremales para problemas parabolicos de evolucion no lineales y aplicaciones, Ph.D. thesis, Departamento de Matemática Aplicada, Universidad Complutense de Madrid, 2005.

J.M. Arrieta and A. Rodríguez-Bernal

Departamento de Matemática Aplicada,

Universidad Complutense de MAdRID, Madrid España .

E-mail address: arrieta@mat.ucm.es, arober@mat.ucm.es

Web page: http://www.mat.ucm.es/ jarrieta

http://www. mat.ucm.es/ arober

J. D. Rossi

Instituto de Matemáticas y Física Fundamental

Consejo Superior de Investigaciones Científicas

Serrano 123, Madrid, Spain,

on leave from Departamento de Matemática, FCEyn UBA (1428)

Buenos Aires, Argentina.

E-mail address: jrossi@dm.uba.ar

Web page: http://mate.dm.uba.ar/ jrossi 